\title{
Performance of a Azimuthally Excited 3D-Printed Resonator for Multi-Pass Spectroscopic Applications
}

\author{
Gordon S. Humphries, Ralf Bauer, and Michael Lengden \\ Centre for Microsystems and Photonics, Department of Electronic and Electrical Engineering \\ University of Strathclyde \\ Glasgow, UK \\ gordon.humphries@strath.ac.uk
}

\begin{abstract}
A design is presented for an acoustically resonant cell, based on the excitation of azimuthal resonances, to enhance the sensitivity of multi-pass photoacoustic spectroscopy. The acoustic cell is fabricated in one part using a photo-polymerization based 3D-Printing technique. Characterization of the cell performance is undertaken using calibrated concentrations of methane. The quality factor $(<76)$ of the cell compares favorably to existing designs based on longitudinal resonances. The minimum detectable normalized noise equivalent absorption coefficient for the cell was measured as: $3.667 \times 10^{-10} \mathrm{Wcm}^{-1} \mathrm{~Hz}^{-1 / 2}$, resulting in a minimum sensitivity for methane of 6 ppm with 700 $s$ of averaging.
\end{abstract}

Keywords-Photoacoustic; Spectroscopy; Laser; 3D-Printing; Gas Detection.

\section{INTRODUCTION}

Since it was first reported by A.G. Bell in 1880 [1] the photoacoustic effect has been studied in detail and developed into an effective, highly sensitive technique for the measurement of trace gases [2]. Photoacoustic spectroscopy (PAS) employs a high intensity modulated light source, generally a laser, to generate an acoustic signal. The acoustic signal is generated due to the thermal release of the energy stored during absorption, and is then measured using a microphone, or transducer, combined with a phase sensitive measurement technique (usually in the form of a lock in amplifier). The acoustic signal can be enhanced by enclosing the sample gas in a sample cell which has an acoustic resonance at the laser modulation frequency [3]. A wide range of resonant cell designs have been investigated [4], aimed at exciting either the longitudinal, azimuthal, or radial modes with the clear majority of designs exciting the longitudinal resonant mode . Previously, we have presented the development of medium to low Q-factor cells which can be rapidly manufactured using a photo-polymerisation based 3D-priniting technique [5], [6].

The magnitude of the pressure change generated by the absorption of the excitation radiation by the target gas, and hence the sensitivity of the PAS measurement is directly proportional to the modulated laser power. Power scaling of near-infrared diode lasers using fibre amplifiers (up to several Watts), used in conjunction with longitudinally resonant cells, has been used to undertake sensitive PAS measurements with detection limits approaching what can be achieved using mechanically resonant techniques such as quartz-enhanced PAS (QE-PAS) [7].
Recently work has been undertaken to exploit the extremely high optical powers which can be generated within a highfinesse optical cavity using relativity modest pump powers [8]. Often a factor which can restrict, or limit, the inclusion of resonant cells within such sensors is the small diameter required to maintain a useful acoustic enhancement. This problem can be overcame by exciting the azimuthal modes of a cylindrical resonator.

\section{Acoustic CEll DesigN}

The 3D-printed resonator developed for this work is shown in Fig. 1 and is a relatively simple design which consists of a single $20 \mathrm{~mm}$ x $40 \mathrm{~mm}$ cylinder, with $6 \mathrm{~mm}$ diameter ports situated off-axis for optical access. Microphones (Knowles, FG-23742-D36) are located at the top and bottom of the cylinder for measurement of the nodes and antinodes of azimuthal resonant modes. The microphones are connected to the internal cylinder by $0.5 \mathrm{~mm}$ long, $0.76 \mathrm{~mm}$ diameter tubes, which match the diameter of the microphone port.

Fabrication of the resonator is completed in a single step using a stereolithography based 3D-Printer (EnvisionTec, Perfactory Desktop Aureus) which has a minimum resolution of $25 \mu \mathrm{m}$. The advantage of using such a high resolution is that the microphone holders can be fabricated with high tolerances, providing a tight seal around the microphone, which is finally sealed using adhesive.

A finite element model (FEM) of the internal structure of the resonator was constructed using Comsol Multiphysics to evaluate the eigenfrequency and pressure distribution of the azimuthal resonant modes. The FEM analysis was undertaken using air as the sample gas, and identified the first two azimuthal modes at eigenfrequencies of $10.48 \mathrm{kHz}$, and 23.08 $\mathrm{kHz}$ respectively. The acoustic pressure distribution of these modes is shown in Fig. 2

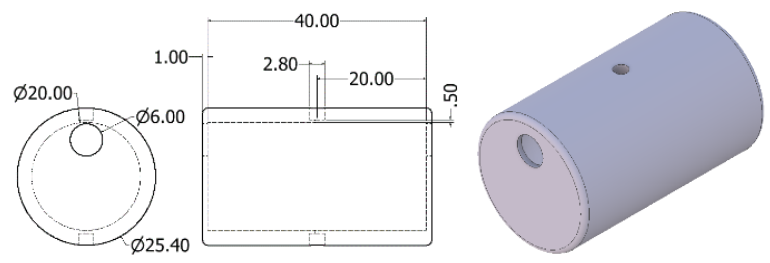

Fig. 1. - Internal and external dimensions of acoustic cell (all sizes in [mm]) 


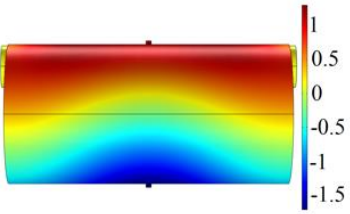

a)

Fig 2. FEM calculated pressure distributions for the first (a) and second (b) azimuthal resonant modes of the acoustic cell.

(labelled mode 1 and mode 2 respectively), and in both the maximum pressure differential is observed between the two microphone ports.

\section{EXPERIMENTAL SETUP}

To allow for control of the gas composition the resonator is enclosed in an aluminum gas cell into which the flow of calibrated test gases is controlled using mass flow controllers (Bronkhorst, EL-Flow). The excitation laser used for the photoacoustic evaluation was a $1653 \mathrm{~nm}$, distributed feedback (DFB) diode laser (OKI) with an average power output of 7.3 $\mathrm{mW}$. Thermal (and hence course wavelength control of the diode) was achieved using a thermos-electric element within the laser package, and external controller (Thorlabs, TED200). The drive current was supplied to the laser using a laser diode controller (Thorlabs, LDC202), the output of which can be controlled externally using an analog voltage. An arbitrary waveform generator was used to create a $44 \mathrm{~mA}$ linear current ramp, which was sufficient to scan the laser wavelength over an absorption feature. The higher frequency modulation required to excite the acoustic resonances of the cell was generated using a sinusoidal output from a second channel from the waveform generator. This higher frequency modulation was applied to the laser diode using a bias-T circuit. Filtering and demodulation of the acoustic signals detected by the microphones was achieved using a Stanford Research SR830 lock in amplifier (LIA), the output of which was recorded using a digital oscilloscope (Tektronix, TDS3000). The LIA used for this work includes a function to measure the differential between two signals and this is used to subtract the signal measured by the bottom microphone from the top microphone before demodulation.

\section{Acoustic Cell Performance}

The performance of the 3D-printed acoustic cell was evaluated using the methane $\left(\mathrm{CH}_{4}\right)$ absorption transition at $1653.95 \mathrm{~nm}$, at calibrated concentrations of $10 \%, 1 \%$, and $1000 \mathrm{ppm}$ in nitrogen $\left(\mathrm{N}_{2}\right)$. The modulation index $m$ (the ratio of the frequency shift caused by the high frequency modulation to the full width half maximum of the absorption feature) was maintained at 2 for all measurements presented. $f$

Fig. 4. and Fig. 5 show the peak magnitude of the LIA output signal as the modulation frequency is changed in small steps $(10-50 \mathrm{~Hz})$ near the eigenfrequencies of the first and second order azimuthal mode respectively, for a methane concentration of $10 \%$. The LIA settings in both cases were an integration time constant setting of $300 \mu$ s, and a sensitivity of $500 \mu \mathrm{V}$. The acoustic enhancement of the first azimuthal mode can be observed at a frequency of $10.67 \mathrm{kHz}$, with a Q-factor of 76.21 , with the second azimuthal mode observed at $23.97 \mathrm{kHz}$ with a Q-factor of 108.93. The difference in resonance frequency compared to the COMSOL model is due to a combination of the difference in the speed of sound in the sample gas, compared to nitrogen, and minor variations on the internal surfaces of the acoustic cell which occurring during fabrication. The reduction in the peak signal which is observed for the second azimuthal mode is due to the distribution of the acoustic pressure across a higher number of nodes.

The excitation of the azimuthal modes provides a much higher Q-factor compared to other 3D-printed cells which are designed for longitudinal excitation [5], [6], for which Qfactors of approximately 15 have been reported. A further advantage of azimuthal excitation is the presence of an antinode at the bottom of the cell. Fig. 5 shows the recovered photoacoustic signal at each microphone and the enhancement which is a result of measuring the differential between the signals, again

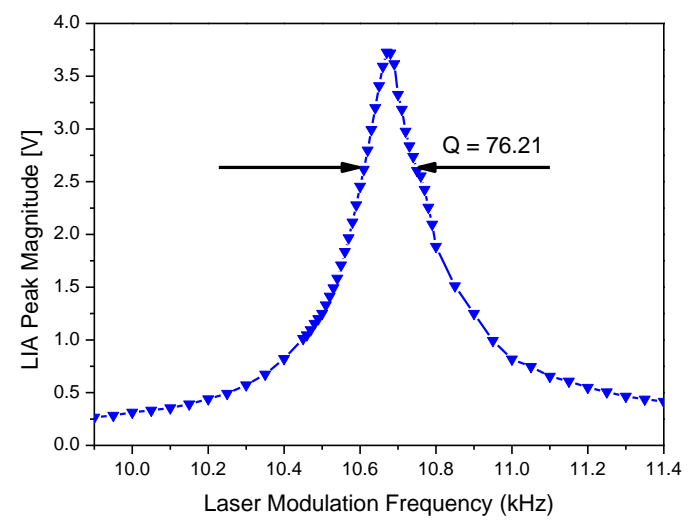

Fig. 3. Measured acoustic frequency response for excitation of the first azimuthal mode at $10.67 \mathrm{kHz}$, when the cell contains $10 \%$ methane in nitrogen.

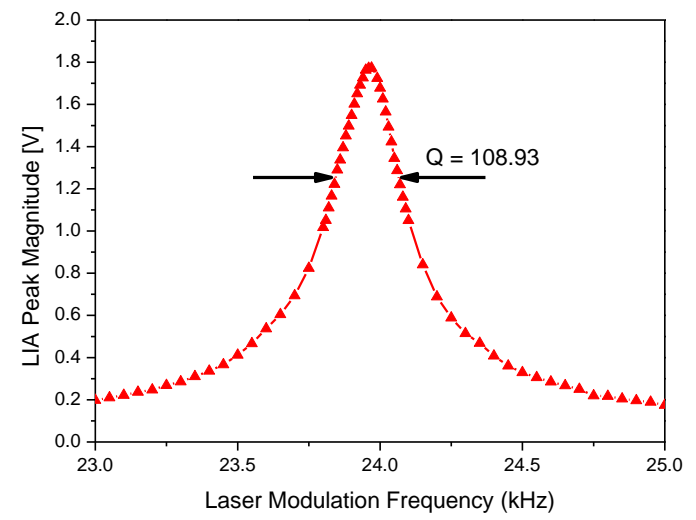

Fig. 4. Measured acoustic frequency response for excitation of the second azimuthal mode at $23.97 \mathrm{kHz}$, when the cell contains $10 \%$ methane in nitrogen. 


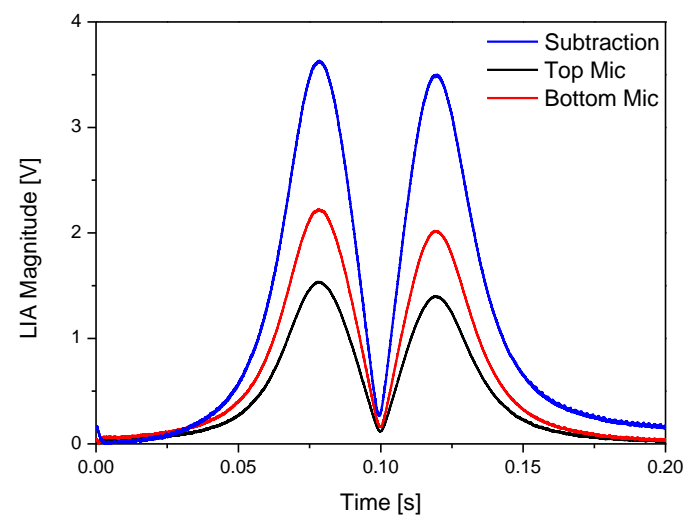

Fig 5. LIA magnitude signals recorded at each microphone, and for predemodulation subtraction for the first azimuthal mode. Signals recorded at $10.67 \mathrm{kHz}$ modulation frequency, with the acoustic cell containing a $10 \%$ methane concentration.

for a methane concentration of $10 \%$. The signal from the microphone located at the antinode is approximately $30 \%$ greater than the signal from the top microphone which agrees with the difference in the acoustic pressure at these points predicted by the COMSOL model.

To evaluate the potential detection limit and long term stability of the acoustic cell the Allan-Werle deviation was calculated as a function of the integration time for each of the two azimuthal modes. The peak LIA magnitude was measured at $0.8 \mathrm{~s}$ intervals for a period of $2 \mathrm{hrs}$., using a LIA integration time of $300 \mu$ s and a sensitivity of $20 \mu \mathrm{V}$. This was undertaken using a methane concentration of $1000 \mathrm{ppm}$ for the first azimuthal mode and using a $1 \%$ concentration for the second mode, the results of the Allan-Werle deviation measurements are shown on Fig. 6. When the first azimuthal mode is excited a detection limit $(3 \sigma)$ of $135 \mathrm{ppm}$ can be achieved without averaging. The Allan-Werle deviation, for the first azimuthal mode reaches a minimum detection limit $(3 \sigma)$ of $6 \mathrm{ppm}$ following $700 \mathrm{~s}$ of averaging. The detection limit $(3 \sigma)$ for the second azimuthal mode without, and following $350 \mathrm{~s}$ of averaging is $828 \mathrm{ppm}$ and $45 \mathrm{ppm}$ respectively. The large increase in the long-term stability of the measurement compared to previous 3D-printed acoustic cells can be attributed to the far greater internal volume of the cell which is afforded by exciting the azimuthal modes. The normalized noise equivalent absorption coefficient can be calculated for each of the two azimuthal modes using the non-averaged detection limit. This was determined to be $3.667 \times 10^{-10} \mathrm{Wcm}^{-}$ ${ }^{1} \mathrm{~Hz}^{-1 / 2}$ and $2.264 \times 10^{-9} \mathrm{Wcm}^{-1} \mathrm{~Hz}^{-1 / 2}$ for the first and second azimuthal modes respectively.

\section{CONCLUSIONS}

The design for a 3D-printed acoustically resonant cell which has sufficiently large optical access to be included in multi-pass absorption measurements has been presented. The design of the cell was targeted at the excitation of azimuthal resonances, and the first two azimuthal resonances were identified through a finite element modelling and experimental investigation. The

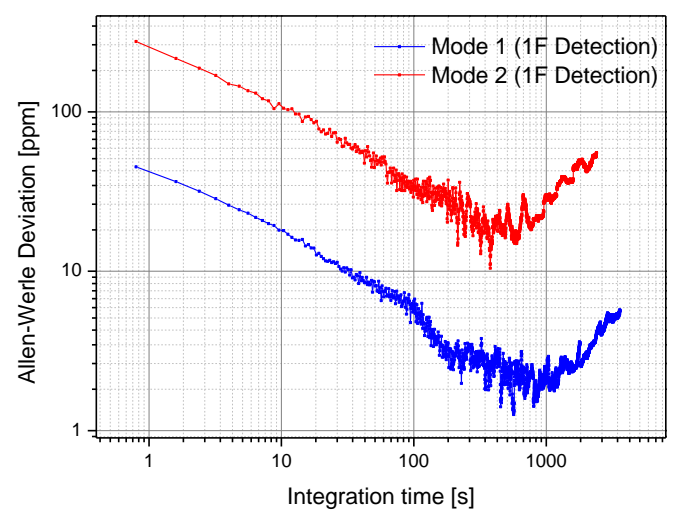

Fig. 6. Allen-Werle Deviation measurements for the first and second azimuthal modes, using a sample gas of $1000 \mathrm{ppm}$ and $10 \%$ methane respectively.

performance of the cell was characterized using an absorption feature of methane at $1653.95 \mathrm{~nm}$. The acoustic cell was found to have a much more favorable Q-factor for the first and second azimuthal modes (76.21, and 108.93 respectively) than has been achieved by longitudinally resonant 3D-printed cells. The minimum NNEA for each mode was $3.667 \times 10^{-10} \mathrm{Wcm}^{-1} \mathrm{~Hz}^{-1 / 2}$ and $2.264 \times 10^{-9} \mathrm{Wcm}^{-1} \mathrm{~Hz}^{-1 / 2}$ respectively which again compares favorably with previous $3 \mathrm{D}$-printed designs. The limit of detection $(3 \sigma)$ for methane was determined to be $6 \mathrm{ppm}$ for first azimuthal mode and $45 \mathrm{ppm}$ for the second mode, following $700 \mathrm{~s}$ and $350 \mathrm{~s}$ of averaging respectively.

\section{REFERENCES}

[1] A. G. Bell, "On the production and reproduction of sound by light," Am. J. Sci., vol. s3-20, no. 118, pp. 305-324, Oct. 1880.

[2] F. J. M. Harren, J. Mandon, and S. M. Cristescu, "Photoacoustic Spectroscopy in Trace Gas Monitoring," in Encyclopedia of Analytical Chemistry, Chichester, UK: John Wiley \& Sons, Ltd, 2012.

[3] P. Hess, "Resonant photoacoustic spectroscopy," Springer, Berlin, Heidelberg, 1983, pp. 1-32.

[4] J. Li, W. Chen, and B. Yu, "Recent Progress on Infrared Photoacoustic Spectroscopy Techniques," Appl. Spectrosc. Rev., vol. 46, no. 6, pp. 440-471, Aug. 2011.

[5] R. Bauer et al., "Miniaturized Photoacoustic Trace Gas Sensing Using a Raman Fiber Amplifier," J. Light. Technol., vol. 33, no. 18, pp. 3773-3780, Sep. 2015.

[6] R. Bauer, G. Stewart, W. Johnstone, E. Boyd, and M. Lengden, "3D-printed miniature gas cell for photoacoustic spectroscopy of trace gases.," Opt. Lett., vol. 39, no. 16, pp. 4796-9, Aug. 2014.

[7] A. A. Kosterev, Y. A. Bakhirkin, R. F. Curl, and F. K. Tittel, "Quartz-enhanced photoacoustic spectroscopy," Opt. Lett., vol. 27, no. 21, p. 1902, Nov. 2002.

[8] A. Kachanov, S. Koulikov, and F. K. Tittel, "Cavityenhanced optical feedback-assisted photo-acoustic spectroscopy with a $10.4 \mu \mathrm{m}$ external cavity quantum cascade laser," Appl. Phys. B, vol. 110, no. 1, pp. 4756, Jan. 2013. 